\title{
Tactile spatial anisotropy with static stimulation
}

\author{
EUGENE C. LECHELT \\ University of Alberta, Edmonton, Alberta, Canada
}

\begin{abstract}
Tactile discrimination of stimulus-orientation discrepancy from standard or referent vertical, horizontal, and diagonal orientations was investigated. A two-alternative forced-choice paradigm was used in which stimuli were presented statically to the distal pads of subjects' right and left index fingers. The results from the present experiment were consistent with previous work employing dynamic tactile stimulation in which a similar and substantial oblique effect was obtained. Specifically, comparison orientations were discriminated more accurately with less deviation from standard vertical and horizontal stimulus orientations than from standard right- and left-diagonal stimulus orientations. The results are discussed in terms of likely underlying sensory mechanisms.
\end{abstract}

Jastrow (1893) was among the first to report perceptual spatial asymmetries in the visual modality. Specifically, he found subjects discriminated stimulus orientations more accurately when they were presented in vertical or horizontal orientations than in oblique orientations. The pervasiveness of this visual "oblique effect" has been documented by Appelle (1972), who reported such spatial anisotropy in animals and humans as well as in children and adults. Explanations for such visual spatial anisotropic occurrences have been provided by Essock (1980) and Appelle and Gravetter (1985). It appears as though both basic sensory and memory factors contribute to such visual perceptual spatial asymmetries.

This oblique effect is not limited to the visual modality, as Lechelt, Eliuk, and Tanne (1976) and Lechelt and Verenka (1980) reported similar spatial asymmetries in the haptic modality as well as in visual/haptic cross-modal discriminations of stimulus orientation. The robustness of this perceptual spatial asymmetry was further extended by Lechelt (1988) when he found a significant tactual oblique effect when stimuli were only presented to the ventral distal portion of the tip of the left index finger via an OPTACON (a reading aid for the blind). Furthermore, nearly identical tactual oblique effects were obtained with sighted, visually impaired, and blind subject groups. The bases for these haptic and tactual anisotropic, oblique effects appear to reflect sensory neurological and experiential factors (see Lechelt, 1988).

As the Lechelt (1988) study employed a dynamic spatiotemporal stimulus presentation with the OPTACON, the present study was conducted to assess if a tactual oblique effect could be obtained on the identical sensory surface area only when using static stimulus presentations. The significance of comparing dynamic and static stimulus presentations would serve to examine the potential differential role of different primary afferent mechanoreceptor units. Previous work by Loomis (1974) and Craig (1980) also

Please direct any correspondence to Eugene C. Lechelt, Department of Psychology, University of Alberta, Edmonton, AB, Canada T6G 2E9. compared static and dynamic tactile discrimination of more complex stimulus configurations.

\section{METHOD}

\section{Subjects}

Five undergraduates, 2 males and 3 females, participated as paid observers.

\begin{abstract}
Apparatus
The subjects were seated comfortably with their hands placed on a Plexiglas plate and their right and left index fingers immobilized in a support that allowed stimuli to be applied to the distal finger pads from below with a constant force and in any orientation.

Two Plexiglas cylinders were machined at one end to a width of $0.38 \mathrm{~cm}$ and a length of $1.88 \mathrm{~cm}$. These surfaces served as stimulus contactors. The other end of each cylinder was rigidly affixed to protractors that permitted the contacting surfaces to be aligned at any specified orientation. Subjects placed their hands on the Plexiglas plate and inserted their right and left index fingers into stabilizing guides that maintained the position of the pads of their index fingertips directly over an opening through which the contactors were electronically activated to make contact for a period of $1 \mathrm{sec}$ at $50 \mathrm{~g}$ of pressure. The vertical orientation of each stimulus contactor impacted along the proximodistal axis of the pads of the index fingertips. Between stimulus presentations, the contactors fell free of the skin so that the next stimulus orientations could be set beneath the fingers.
\end{abstract}

\section{Procedure}

Four stimulus orientations were used as standards: vertical, horizontal, and right and left diagonals. Only one standard orientation was tested during an experimental session. A trial consisted of subjects being presented with two simultaneous pairs of stimulus orientations for $1 \mathrm{sec}$ each, separated by a 2 -sec interpair interval. For all conditions, three of the stimulus contactors were set at a standard orientation and the other was set at one of several comparison stimulus orientations; the position of the latter was randomized and occurred an equal number of times at each of the four possible placements: first set, right or left index fingertip pad; or second set, right or left index fingertip pad.

Four pilot sessions of approximately $30 \mathrm{~min}$ each were conducted with each subject to determine the appropriate deviation from the standard orientations that could be used as comparison stimulus orientations. For vertical and horizontal standards, deviations from $2.5^{\circ}$ to $10^{\circ}$ in either direction from the principal meridians provided for an appropriate distribution of response accuracy, that is, one that ranged from approximately $50 \%$ (chance) to nearly $100 \%$ correct reports. For the standard diagonals, however, deviations had to be increased from $5^{\circ}$ to $20^{\circ}$ to produce an equivalent distribution of response accuracy.

A two-alternative forced-choice paradigm was used in that subjects were asked to report which set of contactors (first or second pair) had 
a comparison orientation that was not the standard orientation for that condition. Several practice trials were provided at the beginning of each experimental session to familiarize subjects with the standard and deviation orientations to be used in that session.

For vertical and horizontal standards, eight comparison orientations were used: $2.5^{\circ}, 5^{\circ}, 7.5^{\circ}$, and $10^{\circ}$ in either direction from true horizontal $\left(180^{\circ}\right)$ and vertical $\left(0^{\circ}\right)$. Similarly, $5^{\circ}, 10^{\circ}, 15^{\circ}$, and $20^{\circ}$ deviations in either direction from the $45^{\circ}$ right and $45^{\circ}$ left (from true vertical) standard diagonal orientations were used. These deviation (comparison) orientations were chosen on the basis of initial pilot sessions, as mentioned, and were tested against each of the four standard orientations.

In an experimental session, each of the eight deviation or comparison orientations were presented once at each of the four possible contactor site (right or left index fingertip) $\times$ stimulus pair (first or second) combinations. Subjects served in each of the four standard orientation conditions 10 times according to a randomized schedule. No feedback was provided.

\section{RESULTS}

As neither the occurrence of the comparison (deviation) orientation at any of the four possible conditions of stimulus pair (first or second set) $\times$ contactor site (right or left index finger) nor the direction of the comparison orientation to either side of the standard orientations yielded any systematic differences in response accuracy, data were combined across these conditions. Also, subjects were nearly identical in their responses across all stimulus conditions. This response homogeneity was not surprising owing to the extensive number of practice trials provided throughout the study. Mean correct reports of which of the two stimulus pairs contained the comparison orienta-

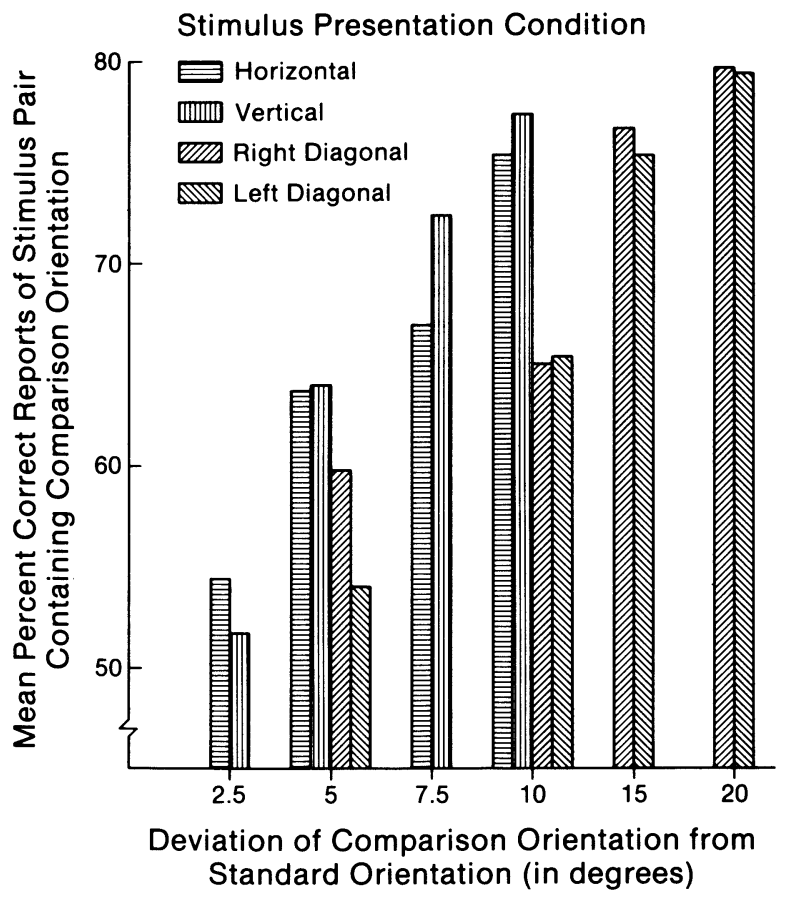

Figure 1. The mean percent correct reports of the stimulus pair containing the comparison orientation shown as a function of the deviation of the comparison orientation from standard horizontal, vertical, right-diagonal, and left-diagonal orientations. tion are thus based on 400 responses (four comparison orientations to either side of each standard $\times 10$ replications $\times$ five subjects).

Using a two-alternative forced-choice procedure, $75 \%$ correct response accuracy is typically considered to define the threshold level of stimulation for correct discrimination; in this instance, for the discrimination of stimulusorientation discrepancy or deviation from the four standard stimulus orientations.

Figure 1 provides an overall summary of the results. It is apparent that accuracy in discriminating stimulusorientation deviation from a standard orientation increases incrementally, and almost linearly, as the magnitude of the deviation increases from $2.5^{\circ}$ to $5^{\circ}$ to $7.5^{\circ}$ to $10^{\circ}$ from the principal vertical and horizontal meridians and, similarly, from $5^{\circ}$ to $10^{\circ}$ to $15^{\circ}$ to $20^{\circ}$ deviations from the right- and left-diagonal standard orientations. Thresholds for the discrimination of stimulus-orientation deviation from vertical and horizontal standard orientations and for either right- or left-diagonal standard orientations are $10^{\circ}$ and $15^{\circ}$, respectively.

\section{DISCUSSION}

The results show a substantial oblique effect in that comparison orientations were discriminated more accurately with less deviation from standard vertical and horizontal stimulus orientations than from standard right- and left-diagonal stimulus orientations. These results obtained with static tactile spatiotemporal stimulation are consistent with those reported by Lechelt (1988) when the same sensory surface was stimulated dynamically, that is, the tactile stimulus orientations were scrolled across the fingertip. Although these studies are not directly comparable because of methodological differences, it is interesting to note that the magnitude of the oblique effect was greater with static than with dynamic tactile stimulation.

The data reported here, along with those of Lechelt (1988), have implications for underlying sensory mechanisms, as well as for the use of the tactile sense in sensory-substitution systems. Phillips and Johnson (1981a, 1981b), in an important series of studies of the neural mechanisms underlying tactile sensory behavior, have documented the relative contributions of three major mechanoreceptor primary afferents: slowly adapting fibers, rapidly adapting fibers, and Pacinian corpuscles. They conclude that, although all major mechanoreceptor units are typically activated upon skin stimulation, the nature of their independent activity is differentially affected by the conditions of stimulation. While slowly adapting mechanoreceptor afferents respond vigorously to dynamic or changing skin stimulation and also with sustained activity to steady indentation, rapidly adapting afferents do not show a sustained response to steady indentation, and Pacinian corpuscles respond uniquely differently to a higher range of temporal modulations than do either slowly or rapidly adapting units. It appears as though slowly adapting fibers have the greatest potential for transmitting the most detailed spatial information about stimulation patterns and are most affected by the orientation of the stimulus on the skin (Phillips and Johnson, 1981b).

Thus, while fundamental peripheral neurophysiological processing would predict superior spatial discrimination under static stimulus conditions that would involve more activity from the slowly adapting fibers, the data from the present study suggest that static tactile stimulus-orientation discrimination is inferior to similar dynamic tactile stimulus-orientation discrimination. The fact that stimuli were presented for only $1 \mathrm{sec}$, however, also makes direct comparisons in terms of differential mechanoreceptor afferent involvement quite tenuous. Regardless, the fact that appreciable and consistent tactile spatial anisotropic effects were obtained under conditions of dynamic and static tactile stimulation discrimination further illustrates the robustness of the oblique effect and its relative independence of the composition of underlying mechanoreceptor activity. 
A comprehensive analysis of perceptual factors associated with static and dynamic tactile stimulus processing can be found in Loomis and Lederman (1986).

Finally, the results have implications for the optimal utilization of the tactile sense in sensory-substitution systems (see Bach-y-Rita, 1980). Gilmore, Hersh, Caramazza, and Griffin (1979) have shown that line orientation is a critical dimension of a stimulus configuration for its discriminability, particularly for letters. Similarly, Kruegar and Ward (1983) have examined Braille reading and conclude that such work must be extended to examinations of how printed material is discriminated at the fingertips. The present results, along with those of Lechelt (1988), at least begin to delineate how one predominant structural feature of letters, namely their intrinsic orientational characteristics, is affected by the specific spatial orientational components of a stimulus pattern, such as a letter, as well as by how such stimulation is presented to the skin (i.e., statically or dynamically). At an applied level, these results have significance for the training of users of the OPTACON as a reading aid of "visual" printed material in that a major factor in letterrecognition errors might well reflect differential tactile resolution of stimulation as a function of inherent orientation components.

\section{REFERENCES}

APPElle, S. (1972). Perception and discrimination as a function of stimulus orientation: The "oblique effect" in man and animals. Psychological Bulletin, 78, 266-278.

APPELle, S., \& GravetTer, F. (1985). Effect of modality-specific experience on visual and haptic judgment of orientation. Perception, 14, 763-773.

BACH-Y-RItA, P. (1980). Sensory substitution in rehabilitation. Sensory World, 39, 12-19.

CRAIG, J. C. (1980). Modes of vibrotactile pattern perception. Journal of Experimental Psychology: Human Perception \& Performance, 6, 151-166.
Essock, E. A. (1980). The oblique effect of stimulus identification considered with respect to two classes of oblique effects. Perception, 9 , $37-46$.

Gilmore, G. C., Hersh, H., Caramazza, A., \& Giffin, J. (1979). Multidimensional letter similarity derived from recognition errors. Perception \& Psychophysics, 25, 425-431.

JASTROW, J. (1893). On the judgment of angles and positions of lines. American Journal of Psychology, 5, 214-248.

KrUegar, L. E., W WARD, M. E. (1983). Letter search by Braille readers: Implications for instruction. Journal of Visual Impairment \& Blindness, 77, 166-169.

LECHELT, E. C. (1988). Spatial asymmetries in tactile discrimination of line orientation: A comparison of the sighted, visually impaired, and blind. Perception, 17, 579-585.

Lechelt, E. C., Eliuk, J., \& TANNe, G. (1976). Perceptual orientational asymmetries: A comparison of visual and haptic space. Perception \& Psychophysics, 20, 463-469.

Lechelt, E. C., VerenKa, A. (1980). Spatial anisotropy in intramodal and cross modal judgments of stimulus orientation: The stability of the oblique effect. Perception, 9, 581-589.

Loomis, J. M. (1974). Tactile letter recognition under different modes of stimulus presentation. Perception \& Psychophysics, 16, 401-408.

Loomis, J. M., Lederman, S. J. (1986). Tactual perception. In K. R. Boff, L. Kaufman, \& J. P. Thomas (Eds.), Handbook of perception and human performance (Vol. 2, chap. 31). New York: Wiley.

Phillips, J. R., \& Johnson, K. D. (1981a). Tactile spatial resolution. II. Neural representation of bars, edges, and gratings in monkey primary afferents. Journal of Neurophysiology, 46, 1192-1203.

Phillips, J. R., \& Johnson, K. D. (1981b). Tactile spatial resolution. III. A continuum mechanics model of skin predicting mechanoreceptor responses to bars, edges, and gratings. Journal of Neurophysiology, 46, 1204-1225.

(Manuscript received October 4, 1991.) 